\title{
A Vesicular Stomatitis Virus-Based Vaccine Carrying Zika Virus Capsid Protein Protects Mice from Viral Infection
}

\author{
Xiaodan Shi ${ }^{1} \cdot$ Jingping $\mathrm{Hu}^{1} \cdot$ Jing Guo ${ }^{1} \cdot$ Chuanjian $\mathrm{Wu}^{1} \cdot$ Sidong Xiong ${ }^{1}$ (D) Chunsheng Dong ${ }^{1}$ (D)
}

Received: 17 September 2018 / Accepted: 14 December 2018/ Published online: 28 February 2019

(c) Wuhan Institute of Virology, CAS 2019

\section{Dear Editor,}

Zika virus (ZIKV) is a mosquito-borne virus that belongs to the Flavivirus family along with dengue virus (DENV), yellow fever virus, West Nile virus, and Japanese encephalitis virus (Ming et al. 2016). ZIKV is a singlestranded positive-sense RNA virus encoding three structural proteins, including nucleocapsid protein $\mathrm{C}, \mathrm{prM} / \mathrm{M}$, envelope glycoprotein $\mathrm{E}$, and seven non-structural proteins. Since 2015, over 70 countries and territories had reported continuing vector-borne transmission of ZIKV, making it a global public health issue. People infected with ZIKV normally have no or mild symptoms that include fever, rash, muscle pain, red eyes, headache, and conjunctivitis. However, a 2015 survey in Brazil found that the number of microcephaly cases dramatically increased, suggesting that ZIKV infection and newborn deformity were closely related (Heukelbach et al. 2016). Additionally, ZIKV infection can cause other severe neurological disorders, such as Guillain-Barré syndrome (Cao-Lormeau et al. 2016). Currently, there is no clinically approved vaccine available.

Recently, application of a series of experimental ZIKV vaccines resulted in reduced viral load in several models of animal infection. These included inactivated virus and plasmid DNA-based vaccines (Abbink et al. 2016; Larocca et al. 2016b), an attenuated live virus based vaccine (Xie et al. 2018), a recombinant adenovirus vector-based

Xiaodan Shi and Jingping Hu have contributed equally to this work.

Electronic supplementary material The online version of this article (https://doi.org/10.1007/s12250-019-00083-7) contains supplementary material, which is available to authorized users.

Sidong Xiong

sdxiong@ @uda.edu.cn

Chunsheng Dong

chunshengdong@suda.edu.cn

1 Jiangsu Key Laboratory of Infection and Immunity, The Institutes of Biology and Medical Sciences, Soochow University, Suzhou 215123, China vaccine (Larocca et al. 2016a; Liu et al. 2018; Xu et al. 2018), and a liposome nanoparticle-based mRNA vaccine (Pardi et al. 2017). Similar to other Flaviviruses, such as DENV, the ZIKV envelope protein $\mathrm{E}$ mediates viral binding to cellular receptors and membrane fusion, and it represents the target for most neutralizing antibodies (Lazear and Diamond 2016). The prM protein typically associates with protein $\mathrm{E}$ to form a heterodimer and is important for proper folding of protein E. Co-expression of prM and $\mathrm{E}$ proteins of several Flaviviruses, including ZIKV, results in secretion of virus-like particles (Boigard et al. 2017). In addition, the mRNA vaccine that encodes the full-length prM and E proteins can resist ZIKV infection (Pardi et al. 2017; Richner et al. 2017). Therefore, prM-E proteins have been the primary targets of most ZIKV vaccine candidates.

Flaviviruses often show antigenic cross-reactivity, which can be beneficial and result in cross-protection. However, humoral cross-reactivity can also exacerbate disease via antibody-dependent enhancement (ADE), of which DENV is the prototypic model (Guzman and Harris 2015). Because ZIKV and DENV share primary vectors (Aedes aegypti and Aedes albopictus for transmission), it is likely that many people infected with ZIKV will be more prone to future infection with DENV serotypes. In such cases, it should be considered whether the presence of a preexisting ZIKV-neutralizing antibody would enhance subsequent DENV infection. This is especially important in vaccine development, because both prM-E and $\mathrm{E}$ antigens represent the primary targets of ZIKV-specific neutralizing-antibody induction, which could possibly result in ADE-related effects. Indeed, a previous study showed that a ZIKV-induced antibody response enhanced DENV serotype 2 replication in vitro (Kawiecki and Christofferson 2016). Therefore, to increase safety, it is necessary to investigate other protective ZIKV antigens, besides prM-E or E proteins, for vaccine development.

The capsid protein plays a crucial role in Flaviviridae biology, with a report indicating that a DENV vaccine 
engineered with a capsid protein alone produced neutralizing-antibody independent immunity and significantly reduced viral loads in the brains of challenged monkeys (Gil et al. 2014). In the present study, a recombinant vesicular stomatitis virus (VSV)-based vaccine carrying the ZIKV capsid protein (VSV-Capsid) was generated. VSV has been shown to be an excellent vector to deliver foreign antigens as a viral vaccine vector (Roberts et al. 1999). Recombinant VSV has been successfully developed for a number of vaccine candidates, such as human immunodeficiency virus (Rose et al. 2001), severe acute respiratory syndrome virus (Kapadia et al. 2005), hepatitis B virus (Cobleigh et al. 2013), influenza virus (Roberts et al. 1998), papillomavirus (Reuter et al. 2002), human respiratory syncytial virus (Kahn et al. 2001), Ebola virus and Marburg virus (Jones et al. 2005; Geisbert et al. 2008). To generate recombinant VSV vaccines, the coding sequence of the ZIKV strain PRVABC59 capsid was amplified by PCR and inserted into VSV backbones between the G-L junction using Nhe I and Xho I restriction enzyme sites (Fig. 1A). Further, these genes were expressed from a single adjacent $3^{\prime}$ promoter as a distinct transcriptional unit on the $G$ and $L$ genes. The recombinant VSV-ZikaE260425 virus expressing the ZIKV E protein DIII domain (E DIII; amino acids 260-425) was used as a parallel control, because ZIKV E DIII is the major antigen region and most specific neutralizing epitopes and E DIII-targeted-antibodies protect mice against lethal infection (Zhao et al. 2016). Recombinant VSVs were recovered through the reverse genetic system in BHK-21 cells, as previously reported (Lawson et al. 1995). Cytopathic changes were observed during the viral-packaging process in infected cells (Supplementary Fig. S1A), and viral titers were determined by $\mathrm{TCID}_{50}$ and calculated using the ReedMuench method. We observed that capsid protein insertion resulted in an approximately tenfold attenuation of VSV replication (Supplementary Fig. S1B). N-terminal flagtagged capsid and ZikaE260-425 protein expression was confirmed by western blot at 12 -h post-infection using antiFlag and anti-E antibodies (Fig. 1A). Immunoblotting further verified capsid and ZikaE260-425 expression in infected cells (Supplementary Fig. S1C).

To assess the efficacy of VSV-Capsid-induced antigenspecific antibodies, 6- to 8-week-old male BALB/c mice were randomly divided into four groups and intranasally immunized with $10^{6}$ PFU VSV-ZikaE260-425 or VSVCapsid, with phosphate-buffered saline (PBS)- or VSVgreen fluorescent protein (GFP)-administered groups used as controls (Supplementary Fig. S2). Antigen-specific antibody immune responses were determined by enzymelinked immunosorbent assay (ELISA) at 1, 3, and 5 weeks post-immunization. Both VSV-Capsid and VSVZikaE260-425 immunization induced high levels of IgG at
3 weeks post-vaccination as compared to control PBS- and VSV-GFP-treated mice. Moreover, higher levels of antigen-specific IgG were observed in VSV-ZikaE260-425immunized mice relative to VSV-Capsid-immunized mice (Fig. 1B). These results indicated that the VSV-Capsid vaccine was capable of inducing a strong ZIKV-specific humoral response comparable to VSV-ZikaE260-425.

The cellular immune response is also important for host protection during infection. Therefore, we monitored ZIKV-specific $\mathrm{T}$ lymphocyte proliferation post-immunization. Splenic lymphocytes from mice at 39 days post-immunization were harvested and seeded in 96-well plates (Corning, NY, USA), followed by stimulation with inactivated ZIKV for $72 \mathrm{~h}$. Significant antigen-specific T cell proliferation was measured by BrdU assay (Roche, Basel, Switzerland), which revealed increased proliferation in VSV-Capsid- and VSV-ZikaE260-425-immunized mice as compared to VSV-GFP-immunized mice (Fig. 1C). To further characterize the cellular responses, we evaluated the ability of splenic lymphocytes to generate interferon (IFN)- $\gamma$ post-immunization. Splenic lymphocytes were harvested and stimulated with inactive ZIKV for $24 \mathrm{~h}$, followed by treatment with propidium monoazide (PMA), inomysine, and bafilomycin A (Sigma-Aldrich, St. Louis, MO, USA) for $6 \mathrm{~h}$ and staining for flow cytometric analysis. VSV-Capsid immunization significantly increased IFN- $\gamma+\mathrm{CD} 8+$, and CD4 $+\mathrm{T}$ cells in immunized mice relative to VSV-GFP-immunized mice, while VSVZikaE260-425 immunization resulted in lower levels relative to VSV-Capsid-immunized mice (Fig. 1D).

To determine whether VSV-Capsid immunization could protect mice from ZIKV infection, we established a ZIKVinfected mouse model. Mice were intraperitoneally infected with $10^{4}$ PFU ZIKV PRVABC59, and the E viral gene level was quantified as an indicator of viral replication in the brain, spleen, blood, spinal cord, and testis of infected mice at different time points. Increased levels of viral replication were observed in the spinal cord and brain at 2 and 4 days post-infection, respectively. Viral replication in the testis was modest relative to the spinal cord and brain at 2 or 4 days post-infection (Supplementary Fig. S2). The immunized mice were subsequently challenged with ZIKV, and quantitative PCR was performed at 4 days post-infection to measure viral replication. As shown in Fig. 1E, VSV-Capsid and VSV-Zika E260-425 immunization, respectively, significantly reduced ZIKV-replication loads in the brain and spinal cord as compared with VSV-GFPimmunized mice. Although there was no statistical difference observed in the testis due to the large variation, we observed reduced viral replication in the testis of VSVCapsid-immunized mice compared to VSV-GFP-immunized mice (Fig. 1E). These results indicated that the VSV- 

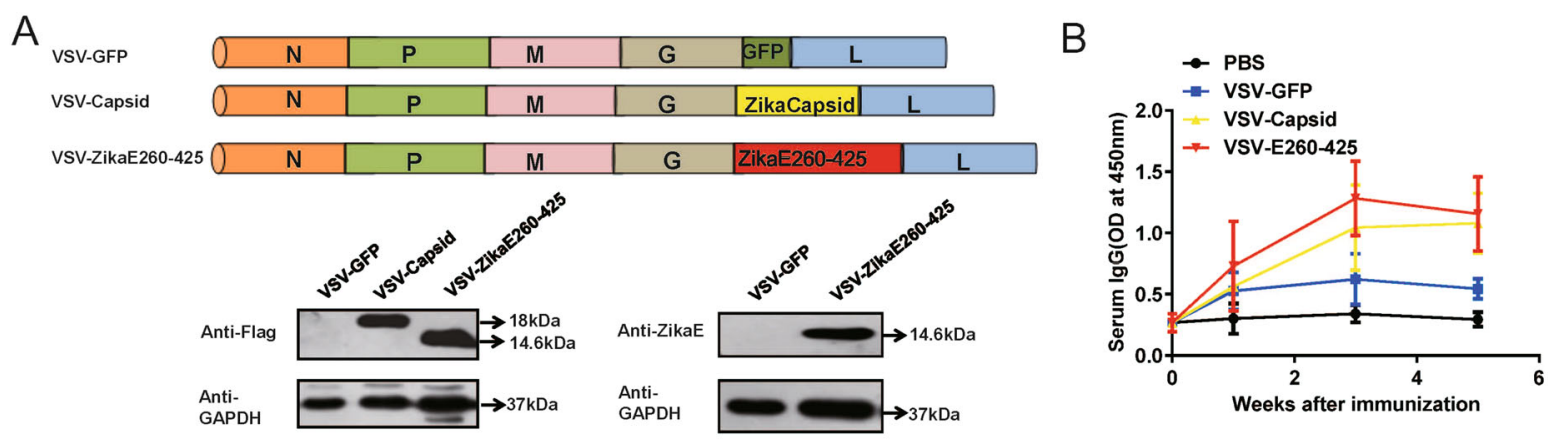

C
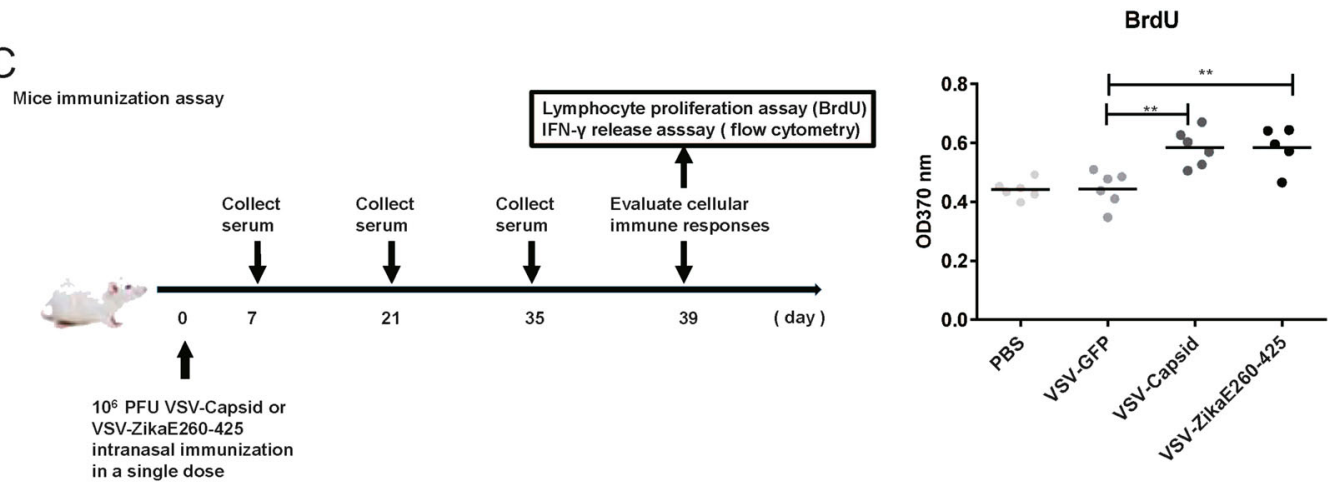

intranasal immuniza

D

Spleen

Spleen
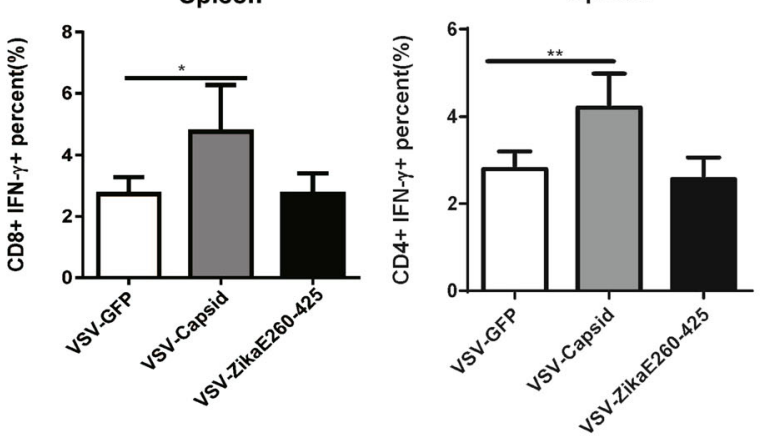

$E$

Mice challenge assay

RT-PCR measure the viral presence in brain, testis and spinal cord of infected mice

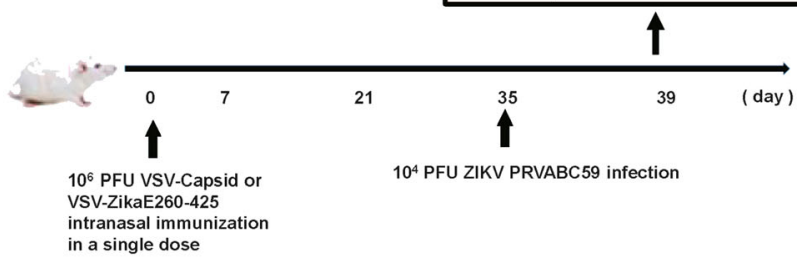

Spinal cord

Brain

Testis
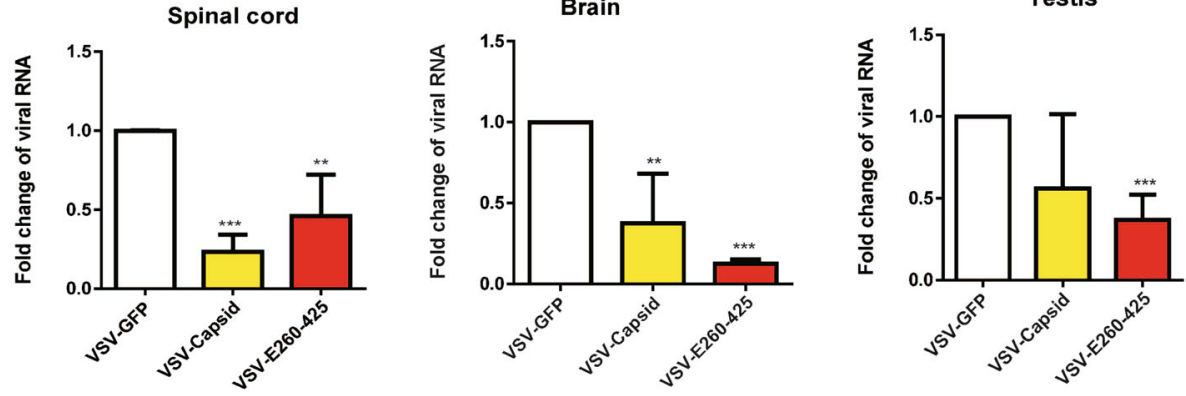
4Fig. 1 Recombinant VSV-ZikaE260-425 and VSV-Capsid vaccines induce immune response and provide specific immune protection in BALB/c mice. A Schematic of the construction of the ZIKV envelope and capsid expression vectors and immunoblot analysis of VSVZikaE260-425 and VSV-Capsid expression in BHK-21 cells infected with recombinant VSVs (10 MOI for $12 \mathrm{~h}$ ) using ZIKV-envelope and Flag antibodies. GAPDH was used as a loading control. B Anti-ZIKV serum titer was analyzed by ELISA using supernatant from Vero cells infected with recombinant VSV-ZIKV $\left(10^{6} \mathrm{PFU}\right)$. C Groups of $\mathrm{BALB} / \mathrm{c}$ mice were vaccinated with $10^{6} \mathrm{PFU}$ VSV-ZikaE260-425 or VSV-Capsid via single intranasal inoculation. Immune responses were detected at the indicated time points. Specific lymphocyte proliferation was detected by BrdU assay in the spleen of mice at 5 weeks post-immunization. D Flow cytometric analysis of antigenspecific IFN- $\gamma$ release from splenocytes in recombinant VSVimmunized $\mathrm{BALB} / \mathrm{c}$ mice after in vitro stimulation with inactivated ZIKV PRVABC59. E Groups of BALB/c mice were vaccinated with $10^{6}$ PFU VSV-ZikaE260-425 or VSV-Capsid via single intranasal inoculation. Five weeks post-immunization, the mice were infected with $10^{4}$ PFU ZIKV PRVABC59, and the viral loads were measured in the brains, spinal cords, and testes of mice immunized 4 days post infection. The relative viral RNA level was normalized with GAPDH gene and set the level in VSV-GFP immunized mice as 1. Error bars indicate SD. $* P<0.05$, $* * P<0.01$, and $* * * P<0.001$, Student's $t$ test.

Capsid vaccine was effective against ZIKV infection following single-dose immunization.

Although the capsid is mainly related to viral replication, studies report that in DENV4, the capsid protein epitope can be recognized by cytotoxic $\mathrm{T}$ lymphocytes, making it a target for antiviral $\mathrm{T}$ cell responses (Gagnon et al. 1996). Additionally, immunization with the capsid alone can induce a protective immune response independent of neutralizing antibodies and primarily dependent upon cell-mediated immunity (Lazo et al. 2007). In the present study, we constructed a recombinant VSV-Capsid vaccine, which resulted in a slightly lower number of generated antibodies than VSV-ZikaE260-425 in immunized mice. This might be attributed to structural features of the capsid, which is not exposed on the cell surface (Teoh et al. 2012). However, in cellular immune response assays, the VSV-Capsid vaccine resulted in elevated specific $\mathrm{T}$ lymphocyte proliferation and IFN $-\gamma$ secretion relative to the VSV-Zika E260-425 vaccine, indicating its potent ability to induce a cellular immune response. Importantly, the viral load was significantly reduced in the brains and spinal cords of challenged mice, indicating the protective role of the VSV-Capsid vaccine in immunized mice against ZIKV infection. It is possible that the VSVCapsid vaccine also protects fetuses in immunized pregnant mice because less virus can cross the placental barrier to establish fetal infection. Moreover, although a VSVbased vaccine VSV-EBOV has been proven safe in trials in Africa and Europe (Agnandji et al. 2016), it is ideal to receive vaccination before pregnancy.
In conclusion, we have developed a novel vaccine expressing ZIKV capsid protein as antigen. Vaccination with VSV-Capsid provided strong immune responses as well as effective protection upon ZIKV challenge in mice. Our findings provide insight into the importance of ZIKV capsid protein for the further development of ZIKVvaccine.

Acknowledgements We gratefully thank Dr. Feifei Yin in Hainan Medical University for providing ZIKV PRVABC59 and also like to thank Prof. Yi Shi in Institute of Microbiology, Chinese Academy of Sciences for providing the Zika E monoantibody. This work was supported by the National Natural Science Foundation of China (31470848, 31470880, 31670898, and 31870867), Open Research Fund Program of the State Key Laboratory of Virology of China (2017IOV003) and Jiangsu Provincial Innovative Research Team.

Author Contributions XS, SX, and CD conceived and designed the research. XS, JH, JG, and CW carried out the experiments. SX and CD analyzed the data. SX and CD wrote the paper. All authors have read and approved the final manuscript.

\section{Compliance with Ethical Standards}

Conflict of interest All the authors declare that they have no conflicts of interest.

Animal and Human Rights Statement All animal experiments were performed in accordance with the guidelines of the Laboratory Animal Ethics Commission of Soochow University.

\section{References}

Abbink P, Larocca RA, Ra DLB, Bricault CA, Moseley ET, Boyd M, Kirilova M, Li Z, Ng'Ang'A D, Nanayakkara O (2016) Protective efficacy of multiple vaccine platforms against Zika virus challenge in rhesus monkeys. Science 353:1129

Agnandji ST, Huttner A, Zinser ME, Njuguna P, Dahlke C, Fernandes JF, Yerly S, Dayer JA, Kraehling V, Kasonta R et al (2016) Phase 1 trials of rVSV Ebola vaccine in Africa and Europe. N Engl J Med 374:1647-1660

Boigard H, Alimova A, Martin GR, Katz A, Gottlieb P, Galarza JM (2017) Zika virus-like particle (VLP) based vaccine. PLoS Negl Trop Dis 11:e0005608

Cao-Lormeau VM, Blake A, Mons S, Lastere S, Roche C, Vanhomwegen J, Dub T, Baudouin L, Teissier A, Larre P et al (2016) Guillain-Barre Syndrome outbreak associated with Zika virus infection in French Polynesia: a case-control study. Lancet 387:1531-1539

Cobleigh MA, Wei X, Robek MD (2013) A vesicular stomatitis virusbased therapeutic vaccine generates a functional CD8 $\mathrm{T}$ cell response to hepatitis $\mathrm{B}$ virus in transgenic mice. J Virol 87:2969-2973

Gagnon SJ, Zeng W, Kurane I, Ennis FA (1996) Identification of two epitopes on the dengue 4 virus capsid protein recognized by a serotypespecific and a panel of serotype-cross-reactive human CD4+ cytotoxic T-lymphocyte clones. J Virol 70:141-147

Geisbert TW, Daddario-Dicaprio KM, Geisbert JB, Reed DS, Feldmann F, Grolla A, Stroher U, Fritz EA, Hensley LE, Jones SM, Feldmann H (2008) Vesicular stomatitis virus-based 
vaccines protect nonhuman primates against aerosol challenge with Ebola and Marburg viruses. Vaccine 26:6894-6900

Gil L, Izquierdo A, Lazo L, Valdes I, Ambala P, Ochola L, Marcos E, Suzarte E, Kariuki T, Guzman G, Guillen G, Hermida L (2014) Capsid protein: evidences about the partial protective role of neutralizing antibody-independent immunity against dengue in monkeys. Virology 456-457:70-76

Guzman MG, Harris E (2015) Dengue. Lancet 385:453-465

Heukelbach J, Alencar CH, Kelvin AA, de Oliveira WK, de Goes Pamplona, Cavalcanti L (2016) Zika virus outbreak in Brazil. J Infect Dev Ctries 10:116-120

Jones SM, Feldmann H, Stroher U, Geisbert JB, Fernando L, Grolla A, Klenk HD, Sullivan NJ, Volchkov VE, Fritz EA, Daddario KM, Hensley LE, Jahrling PB, Geisbert TW (2005) Live attenuated recombinant vaccine protects nonhuman primates against Ebola and Marburg viruses. Nat Med 11:786-790

Kahn JS, Roberts A, Weibel C, Buonocore L, Rose JK (2001) Replication-competent or attenuated, nonpropagating vesicular stomatitis viruses expressing respiratory syncytial virus (RSV) antigens protect mice against RSV challenge. J Virol 75:11079-11087

Kapadia SU, Rose JK, Lamirande E, Vogel L, Subbarao K, Roberts A (2005) Long-term protection from SARS coronavirus infection conferred by a single immunization with an attenuated VSVbased vaccine. Virology 340:174-182

Kawiecki AB, Christofferson RC (2016) Zika virus-induced antibody response enhances dengue virus serotype 2 replication in vitro. J Infect Dis 214:1357-1360

Larocca RA, Abbink P, Peron JP, Zanotto PM, Iampietro MJ, Badamchi-Zadeh A, Boyd M, Ng'ang'a D, Kirilova M, Nityanandam R et al (2016a) Vaccine protection against Zika virus from Brazil. Nature 536:474-478

Larocca RA, Abbink P, Peron JPS, Zanotto PMDA, Iampietro MJ, Badamchi-Zadeh A, Boyd M, Ng'ang'a AD, Kirilova M, Nityanandam R (2016b) Vaccine protection against Zika virus from Brazil. Nature 536:474

Lawson ND, Stillman EA, Whitt MA, Rose JK (1995) Recombinant vesicular stomatitis viruses from DNA. Proc Natl Acad Sci USA 92:4477-4481

Lazear HM, Diamond MS (2016) Zika virus: new clinical syndromes and its emergence in the Western Hemisphere. J Virol 90:4864-4875

Lazo L, Hermida L, Zulueta A, Sanchez J, Lopez C, Silva R, Guillen G, Guzman MG (2007) A recombinant capsid protein from Dengue-2 induces protection in mice against homologous virus. Vaccine 25:1064-1070

Liu X, Qu L, Ye X, Yi C, Zheng X, Hao M, Su W, Yao Z, Chen P, Zhang S, Feng Y, Wang Q, Yan Q, Li P, Li H, Li F, Pan W, Niu X, Xu R, Feng L, Chen L (2018) Incorporation of NS1 and prM/
$\mathrm{M}$ are important to confer effective protection of adenovirusvectored Zika virus vaccine carrying E protein. NPJ Vaccines $3: 29$

Ming GL, Tang H, Song H (2016) Advances in Zika virus research: stem cell models, challenges, and opportunities. Cell Stem Cell 19:690-702

Pardi N, Hogan MJ, Pelc RS, Muramatsu H, Andersen H, DeMaso CR, Dowd KA, Sutherland LL, Scearce RM, Parks R et al (2017) Zika virus protection by a single low-dose nucleoside-modified mRNA vaccination. Nature 543:248-251

Reuter JD, Vivas-Gonzalez BE, Gomez D, Wilson JH, Brandsma JL, Greenstone HL, Rose JK, Roberts A (2002) Intranasal vaccination with a recombinant vesicular stomatitis virus expressing cottontail rabbit papillomavirus L1 protein provides complete protection against papillomavirus-induced disease. J Virol 76:8900-8909

Richner JM, Himansu S, Dowd KA, Butler SL, Salazar V, Fox JM, Julander JG, Tang WW, Shresta S, Pierson TC, Ciaramella G, Diamond MS (2017) Modified mRNA vaccines protect against Zika virus infection. Cell 169:176

Roberts A, Kretzschmar E, Perkins AS, Forman J, Price R, Buonocore L, Kawaoka Y, Rose JK (1998) Vaccination with a recombinant vesicular stomatitis virus expressing an influenza virus hemagglutinin provides complete protection from influenza virus challenge. J Virol 72:4704-4711

Roberts A, Buonocore L, Price R, Forman J, Rose JK (1999) Attenuated vesicular stomatitis viruses as vaccine vectors. J Virol 73:3723-3732

Rose NF, Marx PA, Luckay A, Nixon DF, Moretto WJ, Donahoe SM, Montefiori D, Roberts A, Buonocore L, Rose JK (2001) An effective AIDS vaccine based on live attenuated vesicular stomatitis virus recombinants. Cell 106:539-549

Teoh EP, Kukkaro P, Teo EW, Lim AP, Tan TT et al (2012) The structural basis for serotype specific neutralization of dengue virus by a human antibody. Sci Transl Med 4:139-183

Xie X, Kum DB, Xia H, Luo H, Shan C, Zou J, Muruato AE, Medeiros DBA, Nunes BTD, Dallmeier K et al (2018) A singledose live-attenuated Zika virus vaccine with controlled infection rounds that protects against vertical transmission. Cell Host Microbe 24(487-499):e485

Xu K, Song Y, Dai L, Zhang Y, Lu X, Xie Y, Zhang H, Cheng T, Wang Q, Huang Q, Bi Y, Liu WJ, Liu W, Li X, Qin C, Shi Y, Yan J, Zhou D, Gao GF (2018) Recombinant chimpanzee adenovirus vaccine AdC7-M/E protects against Zika virus infection and testis damage. J Virol 92:e01722-17

Zhao H, Fernandez E, Dowd KA, Speer SD, Platt DJ, Gorman MJ, Govero J, Nelson CA, Pierson TC, Diamond MS, Fremont DH (2016) Structural basis of Zika virus-specific antibody protection. Cell 166:1016-1027 